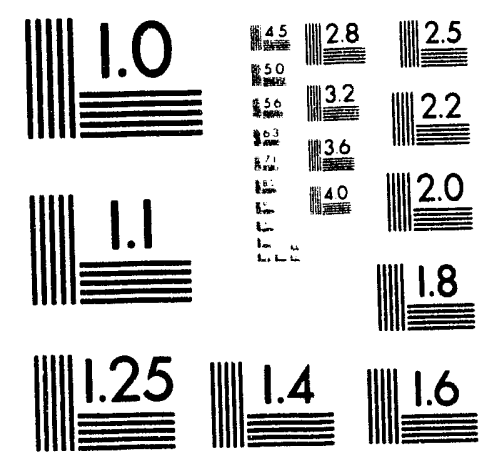



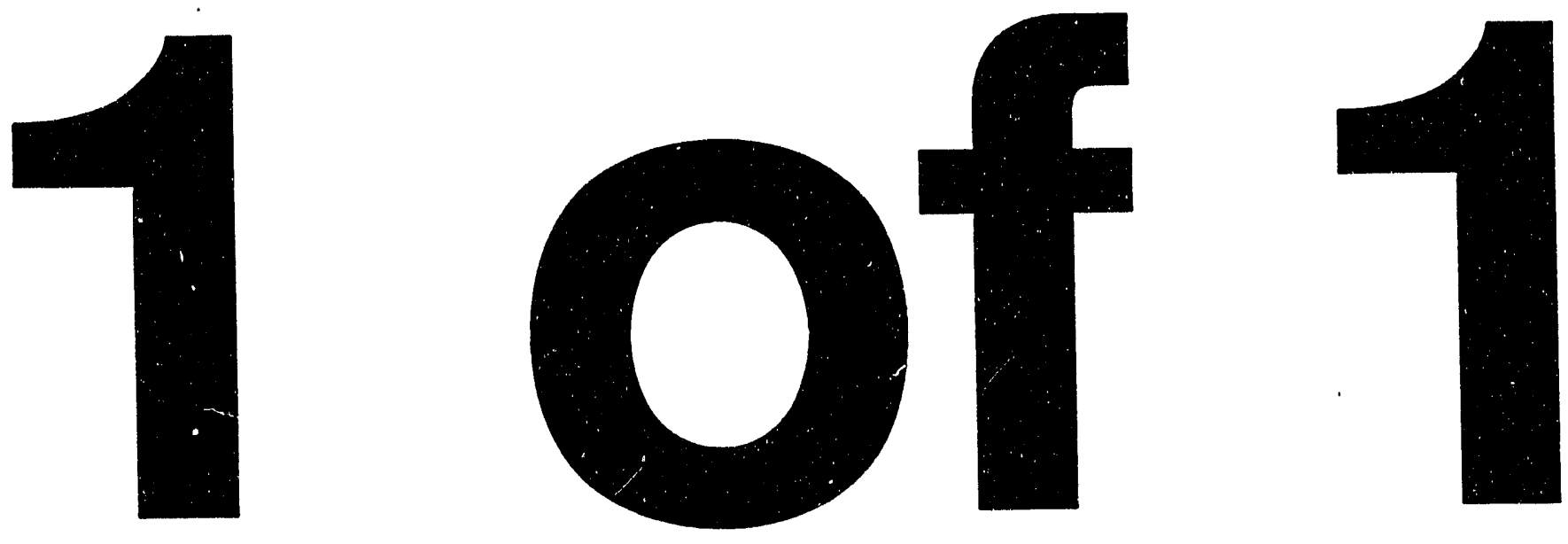


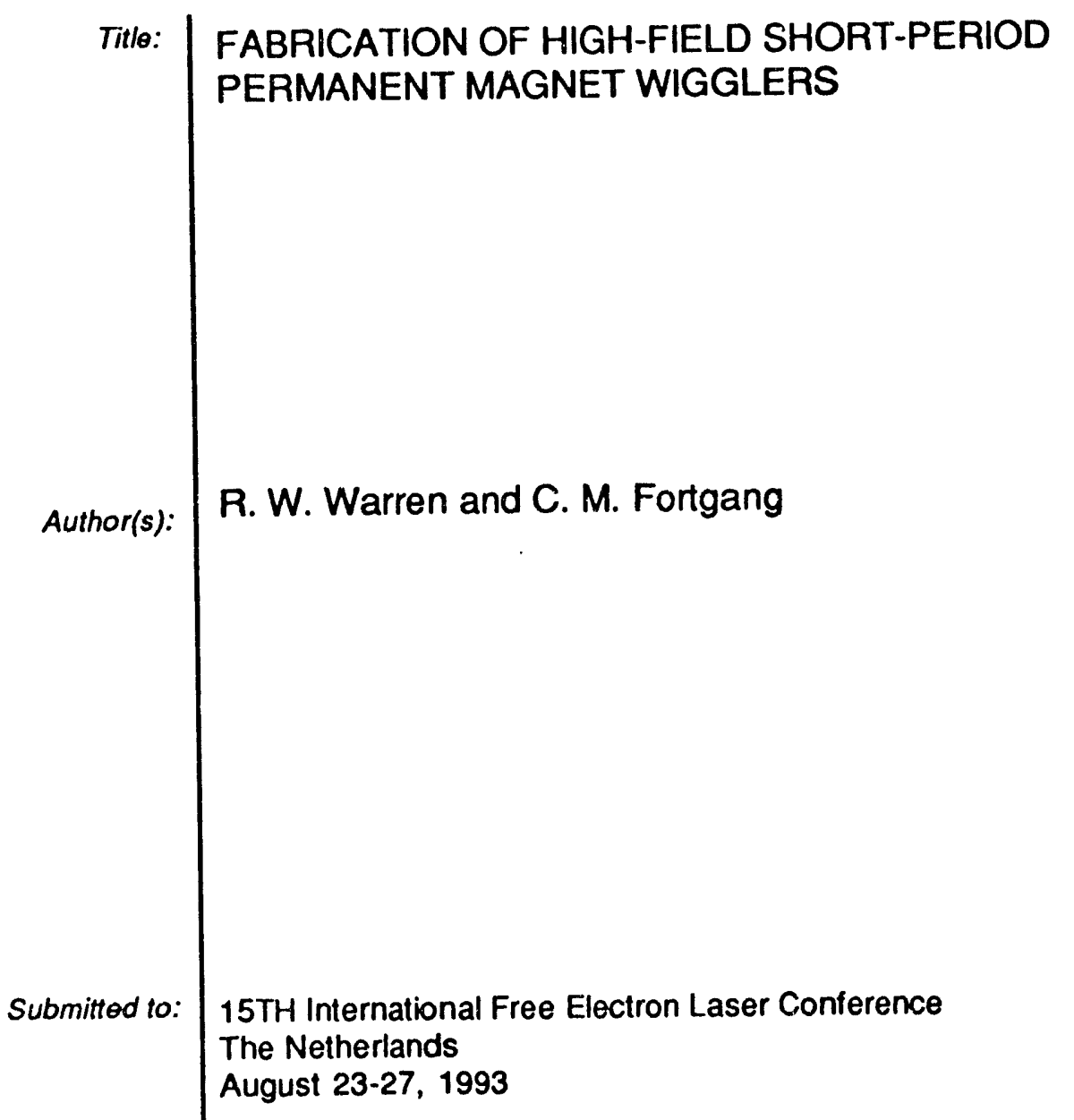

August 23-27, 1993

\section{SEP 07103 \\ OSTI}

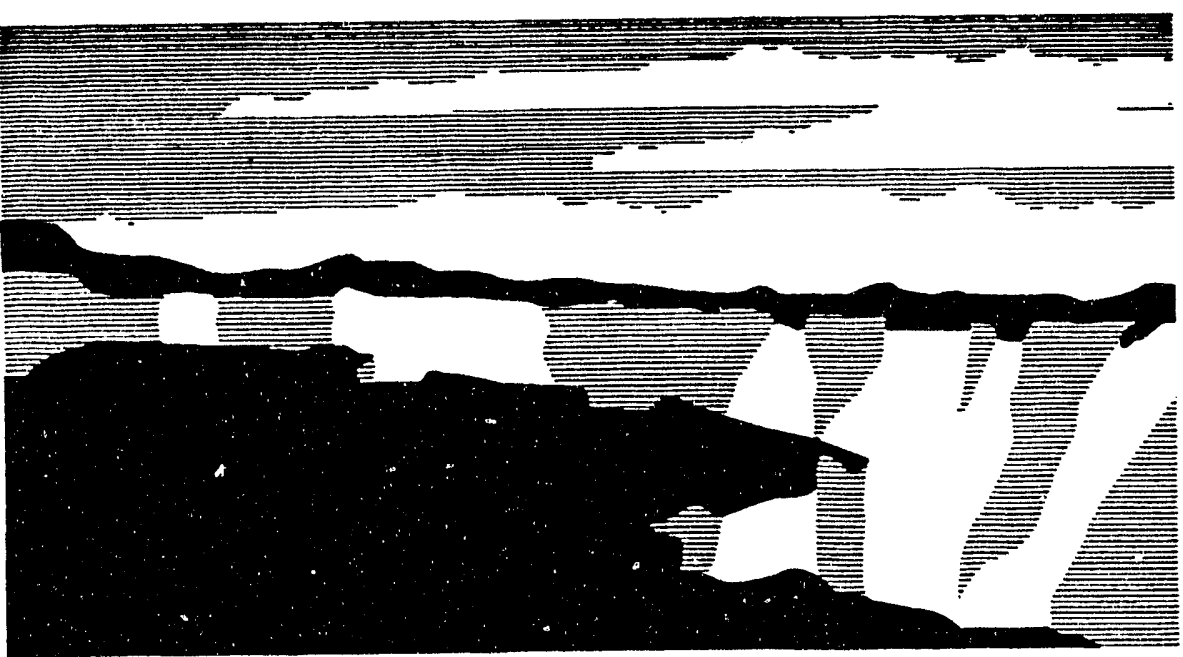
Los Alamos National Laboratory, an aftirmative actionequal opporturity employer, is operated by the University of California tor the U.S. Department of Energy under contract $W-7405-E N G-36$. By acceptance of this article, the publisher recognizes that the U.S. Gover of requests that the publisher identify this article as work performed under the auspices of the U.S. Departinent of Energy. 


\title{
FABRICATION OF HIGH-FIELD SHORT-PERIOD PERMANENT MAGNET WIGGLERS*
}

\author{
R. W. Warren and C. M. Fortgang \\ Mail Stop H825 \\ Los Alamos National Laboratory \\ Los Alamos NM 87545
}

\section{ABSTRACT}

A permanent magnet wiggler is described that has been designed to lase at unusually short wavelengths. Its novel features include the following: all magnets are magnetized parallel to the wiggler's axis; only two pairs per period are used; the gap occupied by the electron beam is very small; the magnet arrangement is optimized for lasing on the third harmonic; the assembly of the magnets is carried out during continuous measurements of the field integrals; field gradients are measured with equal care; and residual errors are corrected by gluing small correcting magnets to appropriate places. The assembly, testing, and trimming of this wiggler was accomplished in less than a week. The wiggler has been used to lase successfully at $0.375 \mu \mathrm{m}$ wavelength.

\section{INTRODUCTION}

Conventional Free Electron Lasers are too large, too expensive, and produce too much hazardous radiation. Minimizing the energy of the accelerator that drives the FEL is the best way to reduce these problems, but maintaining a constant wavelength of light requires either a reduction of the period of the wiggler or lasing on a higher harmonic than the fundamental. Both of these strategies require a wiggler with a large magnetic field. We have previously [1] described our attempts to fashion such a highfield, short-period wiggler using iron-free pulsed electromagnetic wigglers. In this paper we will describe a more conventional approach using a wiggler built of permanent magnets arranged, tested, and adjusted in novel ways.

\section{OUR GOAL}

At Los Alamos, we routinely lase at $\sim 3 \mu \mathrm{m}$ using a conventional permanent magnet wiggler [2] one meter long with a period of $2.73 \mathrm{~cm}$, a peak field of $\sim 0.4 \mathrm{~T}$, using four pairs of magnets per period of square $(0.5$ by $0.5 \mathrm{~cm})$ cross section along with a conventional if accelerator [3] with an energy of $\sim 40 \mathrm{Mev}$, a peak current of several hundred amperes, and very low emittance. We decided to test our accelerator, optical, and wiggler systems by trying to lase at the shortest possible wavelength, limited by the accelerator's maximum energy and modifications we could make to the wiggler using the support structures and magnets we already had.

To accomplish this shift to shorter wavelengths, we increased the accelerator energy to its limit, about $46 \mathrm{Mev}$, cut the wiggler's period in half, and planned to lase on the

\footnotetext{
* Work Supported by the US Department of Energy, office of Defense Programs
} 
third harmonic. The combination of these changes would allow us to lase near 0.37 $\mu \mathrm{m}$ (almost a factor of ten shorter in wavelength than usual) if the gain of the system were large enough. The gain depends primarily upon the electron current, the emittance of the beam, and the field in the wiggler. Calculations showed that the field should exceed $0.5 \mathrm{~T}$ to provide a good chance of lasing.

\section{WIGGLER DESIGN}

Since we were constrained to using existing wiggler structures and existing magnets, the only way to halve the period is to use only two pairs of magnets per period, ieeither of the two arrangements shown in fig.1. An analysis of the field generated by each choice shows that there is a loss of field strength, compared to the usual 4 square magnet pairs/period arrangement, which (for the parameters we used) is only $8 \%$. Either arrangement, shown in fig.1, produces exactly the same strength of the fundamental component as well as all of the harmonics of the field. What differs is the phase relation between the components. When the magnets are arranged as in $1 \mathrm{~A}$, the sum of these components tends to be flat topped, like a square wave. When the magnets are arranged as in $1 \mathrm{~B}$, the sum of the components is sharply peaked. The distortions from a sine wave are fairly small, becoming more extreme as the gap between the upper and lower magnets, as well as the gap between adjacent magnets,

is are reduced. In the limit of small gaps, the strength of the harmonic components varies as the reciprocal of the harmonic number [4].

We have chosen to arrange our wiggler following 1B for three reasons: First, gain on the third harmonic of the fundamental wavelength is derived from two very different mechanisms. The fundamental component of the wiggler's field generates gain on the third harmonic because of the modulation of the fundamental radiation by a slight longitudinal oscillation of the electrons at the second harmonic [5]. The third harmonic component of the wiggler's field generates gain on the third harmonic, by normal processes. These two gain mechanisms add or subtract depending upon their relative phases [6]. For 1B they add; for $1 \mathrm{~A}$ they subtract. Thus the 1B arrangement gives a larger gain for the third harmonic (by about $20 \%$ in our case [7]). Second, with arrangement $1 \mathrm{~B}$ there is no uncompensated angular kick given to the electrons as they enter or leave the wiggler. The third reason for choosing $1 \mathrm{~B}$ is related to our technique for measuring the field, and will be discussed below.

Even with the $1 \mathrm{~B}$ arrangement, the gain is not adequate unless we achieve an unusually large wiggler field. Our only useful strategy is to make the gap between upper and lower magnets very small. Our final choice is $1.5 \mathrm{~mm}$, a compromise between maximizing gain and limiting obstruction of the electron and optical beams. The choice of such a small gap is made possible by experience we have gained in an earlier experiment, passing electron beams down narrow tubes [8].

\section{DEPOLARIZATION EFFECTS}

A disadvantage of the arrangement $1 \mathrm{~B}$ is the possibility of a reduction of the magnetic field, particularly the harmonic components, by a depolarization process. Some of the material in each magnet is operated near the intersection of quadrants 2 and 3 of the $\mathrm{B} / \mathrm{H}$ curve where the field from adjacent magnets tends to reverse the local 
polarization. We built a small model of our wiggler with our standard magnets [9] to test for this possibility but found no discrepancy in field shape or magnitude between the calculated and real models.

\section{ASSEMBLY TECHNIQUE}

The new "miniwiggler" was assembled from parts salvaged from an old conventional wiggler, whose central region is shown in fig.2. The magnets have ends that are cut with orthogonal slots so that a magnet is mounted by meshing its ends with vertical teeth (shown in fig. 1) machined into two bars and is locked in position by inserting two splines into the horizontal slots. The wiggler is then inserted inside the vacuum system. A few supporting parts of the wiggler were remachined to allow the magnets to be arranged with a gap of $1.5 \mathrm{~mm}$. The magnets were reassembled in the new $1 \mathrm{~B}$ arrangement instead of mounting 4 magnet pairs/period. The assembly process was carried out while the field was continually monitored using the pulsed wire technique [10]. This technique has important advantages: it can be used with a small gap, gives rapid results, and allows measurement of quadrupole and higher moment fields. A particular advantage is its use to directly measure the second integral of the field, ie- a quantity that is proportional to the displacement of the electron beam.

Our procedure is to first measure the second field integral when no magnets are in place, as shown in fig.3. Two curves are shown- one is the double integral of By, the "major" wiggle field, and the other the double integral of Bx, the minor field (that ideally would be zero). This measurement gives us a base line, showing the electron displacement caused by the earth's field and by any magnetic hardware in the vicinity. We then insert a pair of magnets, one oriented sideways (to the left) above the axis and the other oriented sideways (to the right) below the axis. Fig.4 shows the displacement generated by this magnet pair (as well as another pair at the other end of the wiggler). The first pair generates a good signal, ie-a stepwise displacement with no slope (compared to the baseline) after the step. The second pair shows a field error, ie-a strong slope after the step. Adding a second opositely oriented pair of magnets causes a displacement in the other direction at a z-shifted position, giving a pulse of displacement with a final displacement of zero and no slope. We keep on adding magnet pairs in this way. Fig.5 shows the displacement with 18 pairs of magnets. Field errors can be easily detected by the existence of a non zero slope or by a non-zero displacement after installing an even number of pairs of magnets. In contrast, if magnet arrangement $1 \mathrm{~A}$ were used, the measurements would have been more complex to interpret and would not have revealed magnet errors with as much sensitivity.

The measuring technique gives electron displacements in both directions perpendicular to the wiggler's axis. Field errors in both directions are revealed and the effects on the electron trajectories are of about the same magnitude. We minimize both errors in two steps: First, we interchange magnets as we assemble them. If, for example, a new magnet pair gives a normal displacement step but an unciesired slope as in fig. 4 above, that pair can be rearranged in a particular way to give the same step but a slope of the opposite sign. Using this observation, a bad magnet pair can be compensated by a new pair, selected and arranged to keep the magnitude of the 
slope below some critical value. This selection process can be used to "steer" the electron beam in any direction desired.

So far we have used this interchange technique mainly to control the errors in By, iein the major component of the field. If both components, Bx and By, were to be corrected, we would have to assemble a large library of magnets with different field characteristics in both $x$ and $y$ to act as compensation for bad magnets. This could be done, but we choose, instead, to correct all of the errors in one plane by selection and rearrangement and to correct the smaller errors in the other plane by a technique [11] that uses very small correcting magnets that are glued onto the outside of magnets near the source of field errors. We are able, in this way, easily to correct for dipole field errors in the $\mathrm{x}$ and $\mathrm{y}$ directions as well as quadrupole fields in these directions.

\section{EVALUATION OF FIELD ERRORS IN THE FINISHED WIGGLER}

The completed wiggler's length is $1 \mathrm{~m}$; there are 73 wiggles; the period is $1.86 \mathrm{~cm}$; the gap is $1.5 \mathrm{~mm}$; and the peak field is $0.65 \mathrm{~T}$. A typical set of measurement made on the finished wiggler is shown in Fig.6. Shown are 6 curves, ie-three pairs, each pair showing the electron's wiggle motion in the $x$ direction and much smaller motion in both the $x$ and $y$ directions caused by error fields. The three pairs of curves differ because of shifts of the measuring wire made in the $x$ direction (away from the axis of the wiggler by zero, $+200 \mu \mathrm{m}$, and $-200 \mu \mathrm{m}$ ). A similar set (not shown) shows the results of similar wire shifts in the y direction. An analysis of these curves reveals the presence and magnitude of error fields of various kinds. The scale drawn on this figure is a calibration of the electron beam displacement calculated for a beam energy of $46 \mathrm{Mev}$ and the measured peak wiggler field of $0.65 \mathrm{~T}$. The calibration is accomplished by calculating the peak to peak displacement of such a beam $(38 \mu \mathrm{m})$ and matching this value to the measured height in fig.6. From this calibration, we can estimate the magnitude of the following obvious wiggler errors in fig.6: the steps in displacement at the beginning and end of the wiggler, the off-axis wandering within the wiggler, and the quadrupole focusing that is illustrated by the convergence of the traces made at different $x$ values. We find that the steps at the ends are small, about $50 \mu \mathrm{m}$; the internal wandering is small, about $15 \mu \mathrm{m}$; and the focusing is weak, with a focal length about five times the wiggler's length.

Field errors can reduce gain by two mechanisms: by reducing the overlap of the electron and optical beams and by shifting the timing of the electrons so that their oscillations become out of phase with the light. The wavelength of light generated by this wiggler is anticipated to be $\sim 0.36 \mu \mathrm{m}$ and the mode should have a Rayleigh length of about $20 \mathrm{~cm}$. Such a beam will have a waist radius in the wiggler of about $150 \mu \mathrm{m}$. Thus one can see that the steps and wandering are significantly smaller than the mode's radius, indicating only a small effect on the gain. Similar calculations have been made for the effect of timing shifts, and again the effects are small.

\section{LASING ATTEMPTS}

Three important additions were made to the modified wiggler before trying to lase: $A$ small, $1 \mathrm{~mm}$-high, OTR screen [12] was built to be inserted in the center of the wiggler to display the electron beam's shape and position, two heavy stainless steel beam- 
stops were placed just upstream of the wiggler magnets to protect them from the electron beam, and two long, 1 mil-thick, nickel foils were attached (by their magnetic attraction) to the surfaces of the wiggler magnets nearest to the electron beam to eliminate wakefield effects caused by the gaps between magnets.

Lasing was achieved at $0.375 \mu \mathrm{m}$, operating on the third harmonic, with a small signal gain of about $10 \%$. The electron and optical bearns passed successfully through the wiggler with little loss. No damage occurred to the magnets. The stability of the electron beam and optical resonator are the limiting features of the FEL system, as might be expected from the small cross section of both optical and electron beams. The wiggler behaved in the expected way with little deterioration in gain caused by wiggler errors. Further details on lasing performance can be found elsewhere in the literature [13].

\section{ACKNOWLEDGMENTS}

Steve Gierman helped to assemble the wiggler and to measure and correct the fields. Lasing was achieved through the efforts of the FEL team mentioned in reference 3.

\section{REFERENCES}

1. Roger Warren, "Progress with the slotted-tube pulsed microwiggler," Nucl.Instr.and Meth A318(1992)789-793.

2. R. W. Warren, C. A. Brau, B. E. Newnam, W. E. Stein, J. G. Winston, and L. M. Young, "Status of the free-electron laser experiment at Los Alamos," Freeelectron generators of Coherent Radiation, Physics of Quantum Electronics, V.8, pg.397, edited by S. F. Jacobs, H. S. Pilloff, M. O. Scully, G. T. Moore, M. Sargent III, and R. Spitzer, publ. by Addison-Wesley 1982.

3. P. G. O'Shea, S. C. Bender, D. A. Byrd, B. E. Carlsten,J. W. Early,

D. W. Feldman, R. B. Feldman, W. J. D. Johnson, A. H. Lumpkin, M. J. Schmitt, R. W. Springer, W. E. Stein, and T. J. Zaugg,

"Initial results from the Los Alamos photoinjector-driven free electron laser," Nucl.Instr.and Meth.A318(1992)52-57.

4. G. Brown, K. Halbach, J. Harris, and H. Winick, "Wiggler and undulator magnetsa review," nucl. instr. and Meth. 208 (1983) 65-77.

5. William B. Colson, "The nonlinear wave equation for higher harmonics in freeelectron lasers," IEEE J.Quantum.Electr. QE-17(1981)1417-1427.

6. M. J. Schmitt and C. J. Elliott, "The effect of harmonic wiggler field components on free-electron laser operation," IEEE J.Quantum Electr. QE-23(1987)1552.

7. Private communication from J. C. Goldstein, Los Alamos National Laboratory.

8. R. W. Warren, P. G. O'Shea, S. C. Bender, B. E. Carlsten, J. W. Early, D. W. Feldman, C. M. Fortgang, J. C. Goldstein, M. J. Schmitt, W. E. Stein, M. D. Wilke, 
T. J. Zaugg, B. E. Newnam, and R. L. Sheffield, "Lasing attempts with a microwiggler on the Los Alamos FEL," to be published in the proceedings of the 14th Intn. FEL Conf. in Kobe, Japan, August 24-28, 1992.

9. Magnets were obtained in several batches over a period of several years from Hitachi Magnetics Corp, Edmore, Michigan. The magnet grade was Hicorex 90B.

10. Daryl W. Preston and Roger W. Warren, "Wiggler field measurements and corrections using the pulsed wire technique," Nucl.Instr. and Meth. A318(1992)794-797.

11. C. M. Fortgang, "Field correction for a one-meter long permanent-magnet wiggler," Proc. 1992 Linear Accelerator Conf., Ottawa, Canada (1992) 169-171.

12. A. H. Lumpkin, W. D. Cornelius, W. E. Stein, R. B. Feldman, K. L. Meier, and L. M. Young, "Electron-beam and high-speed optical diagnostics for the Los Alamos HIBAF facility," Nucl.Instr and Meth. A296(1990)769-775.

13. P. G. O'Shea, S. C. Bender, B. E. Carlsten, J. W. Early, D. W. Feldman, C. M. Fortgang, J. C. Goldstein, B. E. Newnam, M. J. Schmitt, R. L. Sheffield, R. W. Warren, and T. J. Zaugg, "Demonstration of ultra-violet lasing with a low-energy electron beam," the proceedings of the 15th Int. FEL Conf., The Netherlands, August 23, 1993.

\section{FIGURE CAPTIONS}

1. Two alternative magnet arrangements

2. Center of the old wiggler

3. Second integral of $B x$ and By vs. $z$ with no magnets

4. Second integrals with magnet pairs at both ends of the wiggler

5. Second integrals with 18 pairs of magnets

6. Second integrals of finished wiggler. The three relatively smooth curves correspond to the minor field, $\mathrm{Bx}$, at three different $x$ positions. The three highly textured curves correspond to $\mathrm{By}$, at these positions. The resolution of these curves is limited by the digital properties of the oscilloscope. 


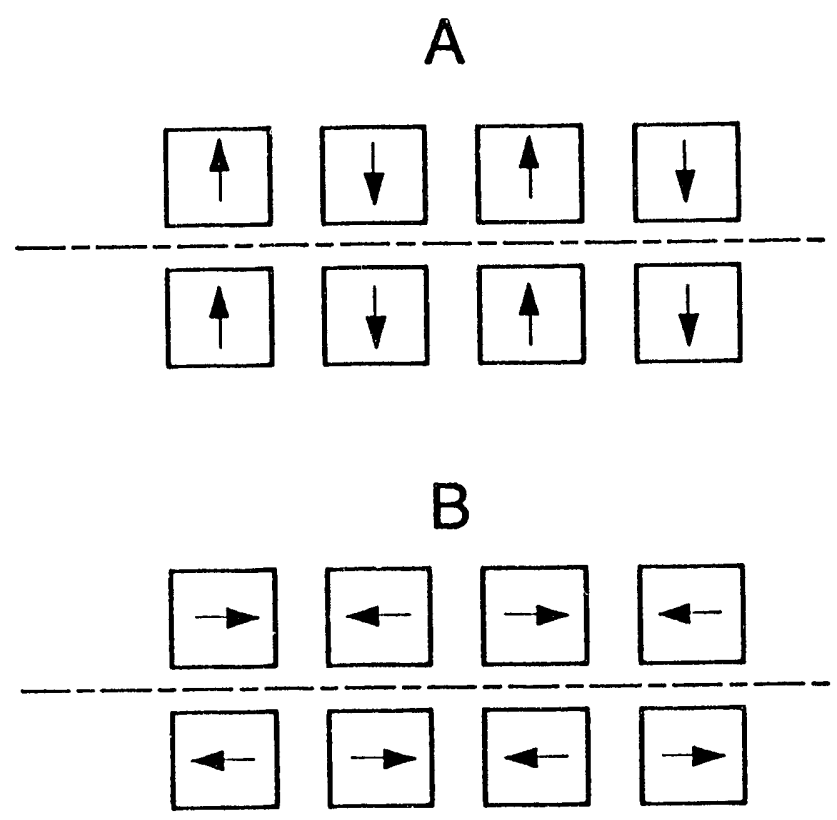

fig 1 


$$
\frac{2}{5}
$$

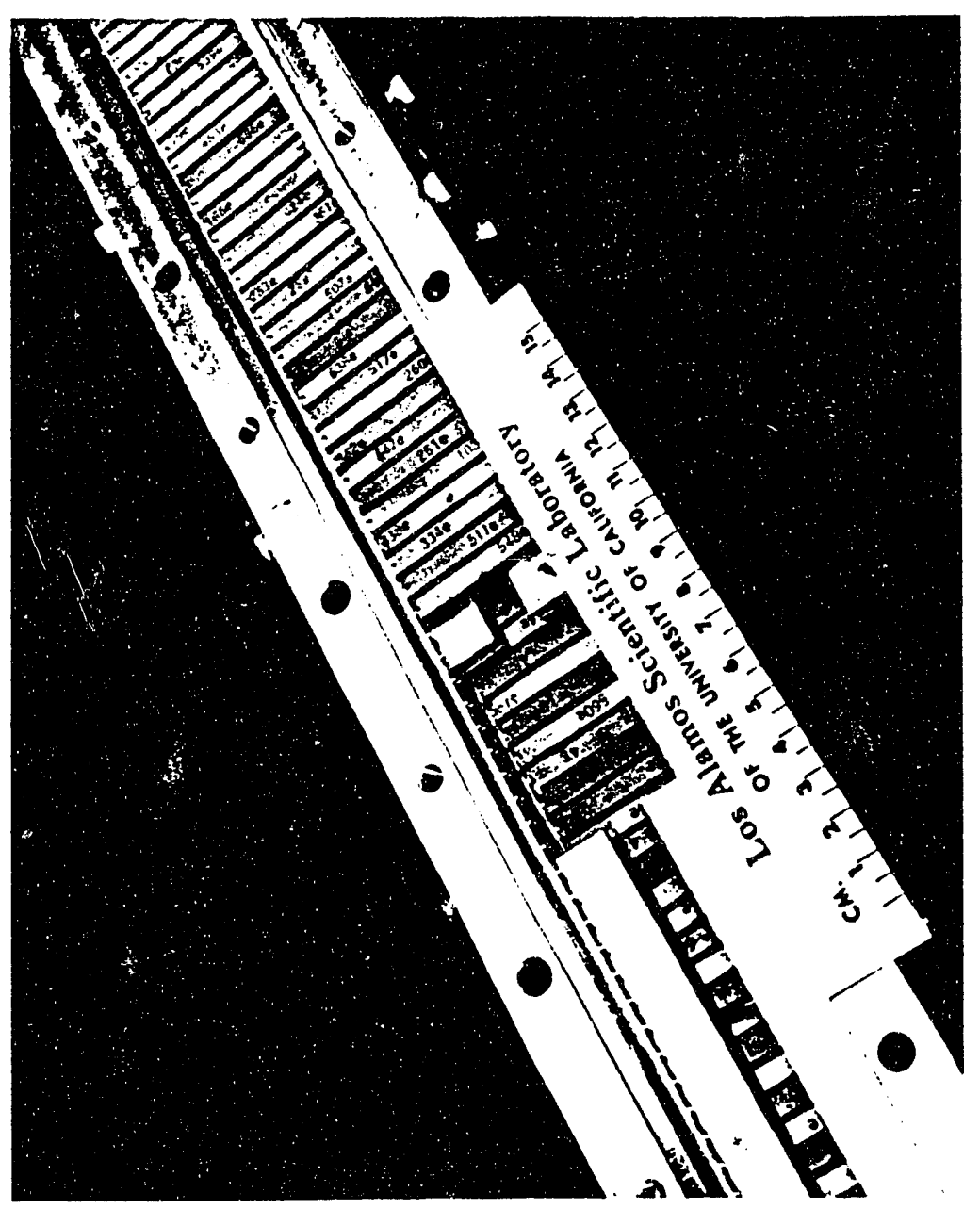




$$
V
$$




$$
\text { V }
$$




$$
\text { I }
$$


6

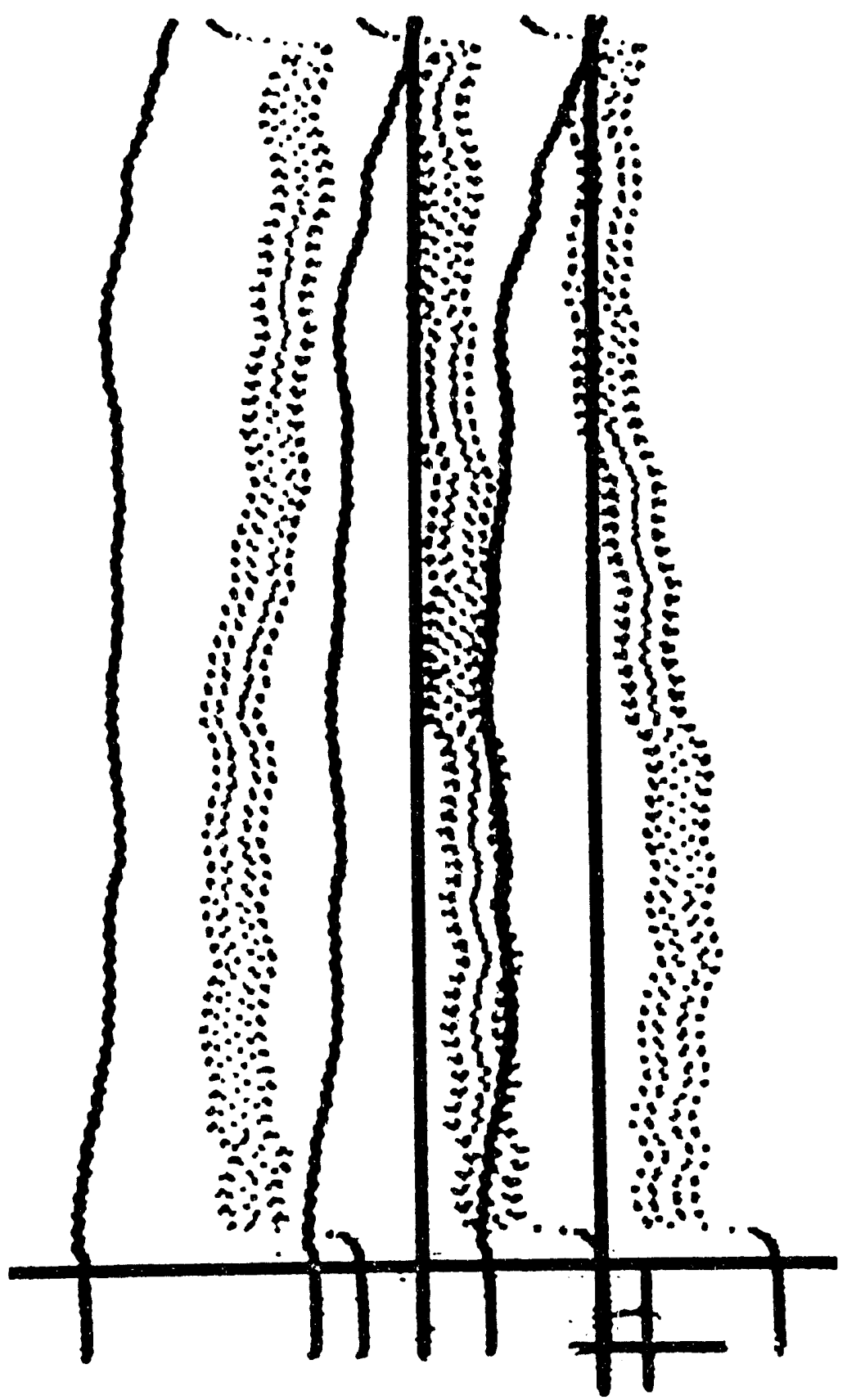



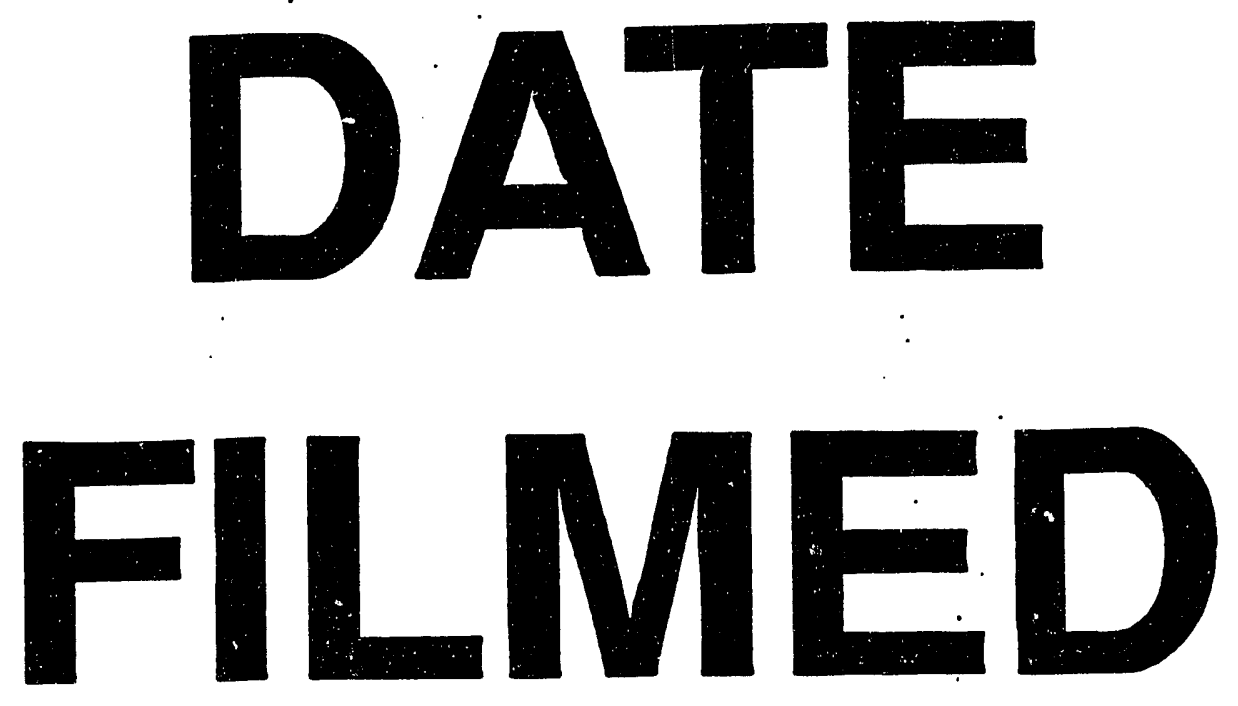

$11 / 4 / 93$
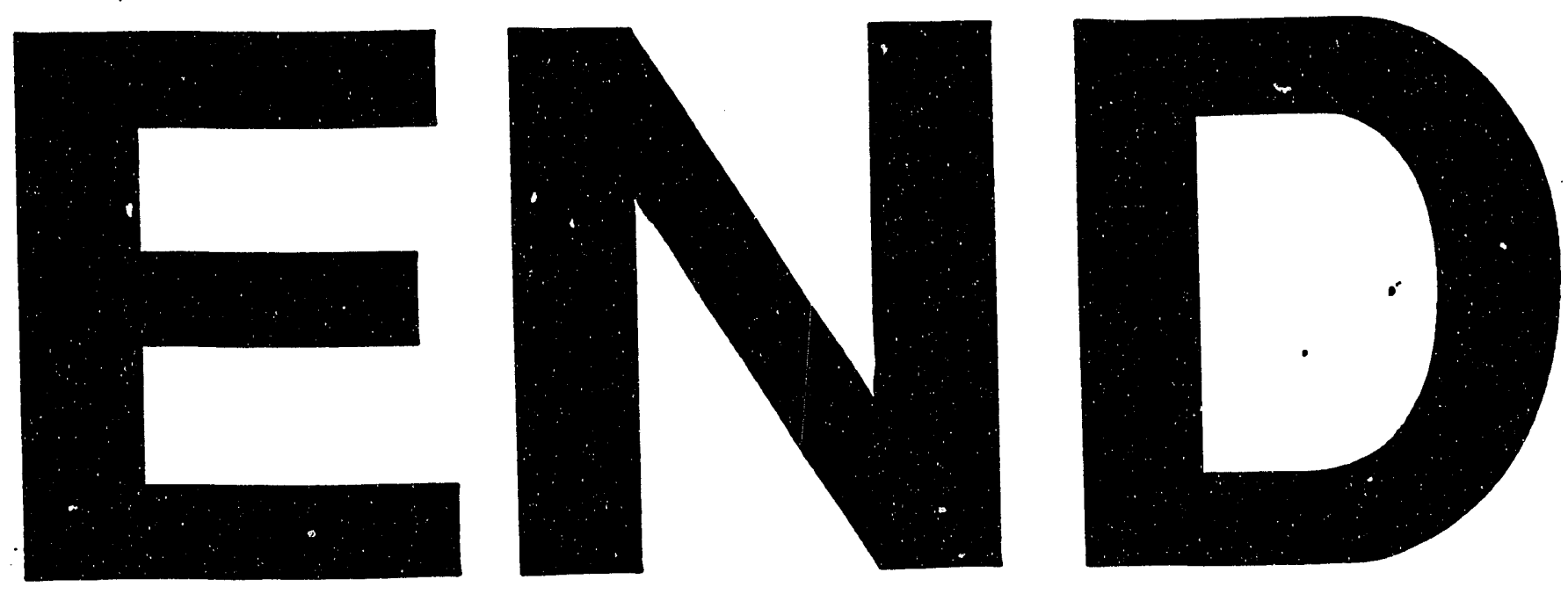
